\title{
Procedural differences in processing intact and degraded stimuli
}

\author{
SANDER A. LOS \\ Vrije Universiteit, De Boelelaan, The Netherlands
}

\begin{abstract}
In three experiments, the extent to which the processing of a visual stimulus profits from equal processing demands of a preceding stimulus was examined. Subjects identified two subsequently presented digits (S1 and S2) that were either intact or degraded by noise, yielding four combinations of stimulus quality. In Experiments 1 and 2, S1 and S2 differed with respect to the values of the digits, so that stimulus quality was the only dimension of possible agreement. The results revealed a faster response to $\mathrm{S} 2$ when the stimulus pairs were homogeneous (both intact or both degraded stimuli) than when they were not homogeneous (degraded-intact pairs and intactdegraded pairs, respectively). The occurrence of equal values of S1 and S2 (Experiment 3) tended to magnify this homogeneous-stimulus effect, but was not a prerequisite for its occurrence. Relative to conditions considered to be neutral, the homogeneous-stimulus effect proved to be due to deviant behavior following the processing of a degraded S1. The suggestion that this reflects the involvement of controlled processing is discussed.
\end{abstract}

A common distinction in the description of human $\operatorname{cog}$ nition is between representation and procedure. It frequently occurs in broad theories in which a representation is conceived as a substrate that results from procedural activity, or on which procedures operate (e.g., Anderson, 1983). On a more basic level, the distinction may set the stage for discussions regarding the nature of various experimental results, namely, whether these originate from the operation of procedures or from the presence or activation of certain representations.

A specific example concerns the controversy regarding the nature of the fast-same effect in sequential matching tasks, in which subjects judge whether two sequentially presented stimuli (S1 and S2) are "same" or “different" (Farell, 1985; Krueger, 1983; Krueger \& Shapiro, 1981b; Proctor 1981; Proctor \& Rao, 1983). The matching response time (T2) is defined as the time elapsing from the presentation of $\mathrm{S} 2$ until completion of the response. The fast-same effect refers to the common observation that $\mathrm{T} 2$ is shorter on same trials than on different trials (e.g., Bamber, 1969; Krueger, 1978; Proctor, 1981). Contrary to the common belief that this effect occurs at the level of matching, for instance, due to differences in time to compare or recheck (Krueger, 1978) representations of same and different'stimuli, Proctor (1981) proposed that the process of encoding is completed faster if a stimulus is preceded by a stimulus that has an identical form. The empirical basis for this encoding facili-

The author is grateful to David E. Irwin, Andries F. Sanders, Gordon D. Logan, and two anonymous reviewers for invaluable comments on earlier drafts of this paper. Correspondence should be addressed to S. Los, Department of Psychology, Vrije Universiteit, De Boelelaan $1111,1081 \mathrm{HV}$ Amsterdam, The Netherlands.

-Accepted by previous editor, Margaret Jean Intons-Peterson tation derives from the observation that the fast-same effect has about the same size in a naming task, in which S2 must be named instead of matched with S1 (Proctor, 1981). Farell (1985) questioned Proctor's implicit claim that matching did not occur in his naming task, but admitted that encoding facilitation is an entirely reasonable construct. Krueger and Shapiro (1981b) argued further that stimulus repetition may entail a shift in criterion rather than increased sensitivity, but Krueger (1983) disconfirmed this view when he failed to find differences between error distributions in a simultaneous and a sequential matching task, thus rendering support for the encoding facilitation hypothesis.

It should be noted that an explanation of the fast-same effect in terms of either matching or encoding facilitation assumes the physical identity of S1 and S2. Nickerson (1975) confirmed the relevance of stimulus identity in a letter-matching task, in which the letters were degraded by various noise patterns. The noise was irrelevant to the task in that the matching response was made with respect to the value of the letters and not with respect to the noise patterns. However, Nickerson found that T2 was shorter when the noise patterns corresponded than when they did not. This result, though interesting in itself, failed to critically contribute to the discussion on the nature of the fast-same effect, because it stands open to several interpretations. Nickerson concluded from his data that all features, whether relevant or not, are represented in memory, and that the comparison of representations evolves faster if S1 and S2 have identical features. Proctor (1981), by contrast, preferred an encoding locus for this effect, arguing that the encoding process is facilitated in the case of stimulus identity.

From a theoretical point of view, it seems more fertile to focus on departures from stimulus identity as a neces- 
sary condition for performance enhancement. In this respect, the empirical data obtained by Hansen and Sanders (1988, Experiment 1) are of interest. They had subjects match two digits that were sequentially presented in the visual field. Either digit was intact or degraded by noise, constituting four conditions of S1-S2 quality: intact-intact, intact-degraded, degraded-intact, and degraded-degraded. The data showed that $\mathrm{T} 2$ was not only affected by the quality of $S 2$, but also by the relation of the qualities of $S 1$ and S2. Specifically, if S2 was intact, T2 was shorter when S1 was also intact than when S1 was degraded, whereas the opposite was true if $\mathbf{S} 2$ was degraded (i.e., T2 was longer when S1 was intact than when S1 was degraded). Because this effect reflects an advantage of processing homogeneous stimulus pairs, it will be denoted here as the homogeneous-stimulus effect (HSE). In line with Nickerson's (1975) account, Hansen and Sanders assumed that their HSE reflected a more efficient matching process in the case of identical stimulus representations. On close inspection, the data do not fully support this interpretation, however. First, Hansen and Sanders used three different noise patterns for each degraded digit, so it is doubtful whether, in a physical sense, the majority of degraded same pairs was any more similar than same pairs in which one of the stimuli was degraded and the other intact. Second, the HSE was, although attenuated relative to same trials, still present on different trials, in which, apart from the digits, the noise patterns were always different. Neither result is predicted by a matching account, which heavily relies on the identity of the stimuli.

At first sight, it seems that an account of Hansen and Sanders's (1988) data in terms of a recapitulation of encoding processes encounters the same problems as does the account based on representations. However, encoding recapitulation makes it easier to bypass the problem of stimulus identity by postulating a process that operates on the noise in a more abstract way, independent of its specific physical structure. Thus, Van Duren and Sanders (1988) suggested that a degraded stimulus needs a process that separates relevant from irrelevant features for its encoding, whereas a fast route, bypassing this process, may be used for encoding an intact stimulus. Consequently, by processing stimulus pairs of the same visual quality, a processing route is opened by $\mathrm{S} 1$, which may facilitate the processing of $S 2$ along the same route. By contrast, processing stimuli of different visual qualities requires a less efficient, and possibly time-consuming switch of processing route. The important feature of this explanation is that it does not exclusively rely on the specific shape of the stimuli, but rather on their molar structure, demanding a specific perceptual analysis.

The proposed account for the HSE in terms of recapitulation of processes relates well to the procedural view of the human mind, which has an important status in research on implicit memory. The basic claim of this view is that our knowledge can be better described in terms of actions than in terms of static representations, such as propositions (Kolers \& Roediger, 1984). Knowledge, it is argued, is not a structure of mere results that is stored in memory, but is inseparable from the way we obtained it (Kolers, 1976; Kolers \& Roediger, 1984). A main source of evidence for this view relies on the dependence of performance on specific stimulus conditions that are very similar to the HSE described here. For instance, Roediger and Blaxton (1987, Experiment 3) had subjects study words that were presented either clearly focused or moderately blurred. In a test episode, the subjects' task was to complete words from which some letters were omitted (e.g., -YS-E-Y for mystery), again presented either blurred or clearly focused. Half of the test words were studied previously and, of these, half were presented in the same stimulus quality as in the study episode, while the other half was presented in the alternate stimulus quality. The data showed a clear advantage of studied words over nonstudied words and, for the studied words, an interaction between the effects of stimulus quality in the study and test episodes. Words studied in clear focus were better completed when they recurred in the test episode in clear focus rather than blurred, whereas the opposite was true for the blurred words. This HSE was regarded as evidence for a closer recapitulation of encoding processes in the case of identical stimulus qualities in the study and test episodes.

\section{Research Questions and Experimental Approach}

Three questions are addressed in the present study. The first and foremost question is whether evidence can be found for a procedural account for the HSE reported by Hansen and Sanders (1988). The experimental approach to this question basically relies on depriving alternative representation accounts from any possible basis, so as to arrive at a procedural account by exclusion. For this purpose, Hansen and Sanders's four stimulus conditions were used, but the experimental design was adapted so that the overlap of actions with respect to $\mathbf{S} 1$ and $\mathbf{S} 2$ was restricted, as much as possible, to encoding processes. To achieve this, three measures were taken. First, following Hansen and Sanders, the functional visual field paradigm (Sanders, 1963) was employed. This experimental paradigm requires different responses to $\mathrm{S} 1$ and $\mathrm{S} 2-\mathrm{a}$ saccade and a manual keypress, respectively. This precludes a priori any overlap of response or response-selection processes, and thus any tendency of the subject to repeat a previous response (see also Krueger \& Shapiro, 1981a). Second, instead of a matching task, a task that requires independent responses to $S 1$ and $\$ 2$ was used, reducing the possibility that matching would be the locus of the HSE. Third, the possibility of matching was further reduced in the first two experiments, in which $\mathbf{S} 1$ and $\mathbf{S} 2$ represented both different digits and noise patterns, rendering stimulus quality as the only dimension of possible agreement. Only in Experiment 3 were conditions added in which S1 and $\mathbf{S} 2$ represented identical digits and noise patterns, in order to establish whether stimulus identity would further add to the HSE.

The second question is whether the HSE as a procedural phenomenon relies on a data-driven or a strategic mechanism. The proposed procedural account relies on a data- 
driven mechanism, in that it assumes that the HSE on T2 is caused by the act of processing S1. That is, subjects are fast in processing $\mathbf{S} 2$ of homogeneous stimulus pairs (or, alternatively, slow in processing $\mathbf{S} 2$ of heterogeneous stimulus pairs) because they engaged in similar (respectively, dissimilar) processing activity for the benefit of S1. In this respect, the mechanism differs from the original proposal of Van Duren and Sanders (1988), which is strategic in that it assumes a preparatory activity on the part of the subject. The preparation implies the advance selection of a processing route for the "worst case" expected on a trial. According to this principle, the slow route is selected in advance whenever a degraded stimulus on a trial is expected, whereas the fast route is only selected when both $\mathbf{S} 1$ and $\mathbf{S} 2$ are expected to be intact. Thus, the processing of intact stimuli of heterogeneous pairs evolves relatively slowly, because it takes place (in part) along the suboptimal slow route. In Hansen and Sanders's (1988) study, such a preparatory mechanism might have played a role, because the specific S1-S2 pairs were presented in pure blocks of trials, which allowed their subjects to select the fast processing route in advance of a trial in the intact-intact condition. In order to establish the contribution of this strategic mechanism to the HSE, a "blocked" and a "mixed" presentation of intact and degraded S2s were compared in Experiment 1. The absence of advance knowledge on the forthcoming S2 in the mixed condition should, according to the worst case principle, provoke the selection of the slow route in any condition. Consequently, the HSE should at least be reduced in the mixed condition relative to the blocked condition if it relies on the strategic principle proposed by Van Duren and Sanders, but not if it relies on a data-driven principle.

The third and final question is whether the HSE is facilitating or inhibiting in nature. Hansen and Sanders (1988) tacitly assumed facilitation to be the driving force behind their effect, but inhibition constitutes a perfectly equivalent alternative. That is, instead of being advantageous to repeat a same route, it could very well be disadvantageous to have to switch from one route to another. The problem of deciding between facilitation and inhibition is that it depends on a control condition, whose neutrality is not easy to defend (e.g., Jonides \& Mack, 1984). In the present study, a control condition was used in which an auditory S1 was combined with an intact or degraded S2. The virtues of this control condition are threefold. First, it allows the employment of essentially the same task as in the experimental conditions (which is not the case if $\$ 2$ is presented in isolation). Second, the information value of the tone can be easily equated to that of a visual S1 by varying the pitch of the signal. Third, it avoids the engagement into visual processing before $S 2$ is presented, which is obviously desirable given the purpose of the present study. There are, however, at least two possible objections to this control condition. First, contrary to a visual S1, an auditory S1 may tend to alert subjects (Niemi, 1979; Sanders, 1977), thus shortening the subsequent T2. Second, it is unclear whether costs are involved in changing from auditory to visual processing, thus prolonging the subsequent $\mathrm{T} 2$. In the General Discussion, the seriousness of these objections will be commented upon, and an evaluation of the neutrality of the control condition will take place in the light of the data obtained. For the present, however, the control condition will be referred to as auditory, rather than neutral.

\section{EXPERIMENT 1}

Experiment 1 was an attempt to acquire insight into the three research questions introduced above. First, it was a test of whether an HSE would occur if stimulus quality is the only common factor between S1 and S2. Second, it was an attempt to establish the strategic contribution to the HSE by comparing conditions in which intact and degraded S2s were presented in either pure or mixed blocks of trials. Third, it was an attempt to establish whether the HSE is due to facilitation or inhibition, by comparing it with the auditory conditions.

\section{Method}

Subjects. The subjects were 24 students, 12 in either of two groups. Six subjects of either group were female. All the subjects had normal or corrected-to-normal vision. They were paid for their services.

Apparatus. The experiment took place in a sound-attenuated, air conditioned, dimly illuminated cubicle. The subject was seated in front of a table, on which a semicircular, 50-cm-high black screen was mounted, which was enclosed by a black ceiling and bottom. The subject had his/her head on a chinrest, positioned $10 \mathrm{~cm}$ above the bottom and at a distance of $70 \mathrm{~cm}$ from the screen. The screen had two windows at eye level and at either side of the visual meridian, subtending a binocular visual field of $45^{\circ}$. The windows were covered with translucent paper, on which the visual stimuli were projected from behind by means of a Kodak carousel projector. Auditory stimuli were presented via padded earphones, which the subject wore during an experimental session. Horizontal saccadic eye movements were registered by $\mathrm{AgCl}$ electrodes, yielding a standard electrooculogram (EOG), which was sampled with a frequency of $250 \mathrm{~Hz}$. On the table, $20 \mathrm{~cm}$ below the bottom of the screen, a response panel was mounted in front of the subject. The panel consisted of two keys, on top of which the subject rested the left and right index finger. An Olivetti M280 microcomputer controlled the timing of events and stored the sampled EOG data and reaction times.

Stimuli. Auditory and visual stimuli were used. The auditory stimuli were binaural 70 -dBA tones of either low $(500 \mathrm{~Hz})$ or high $(1000 \mathrm{~Hz})$ frequency. The visual stimuli were very similar to the ones used by Hansen and Sanders (1988), and consisted of intact and degraded qualities of the digits $2,3,4$, and 5 (see examples in Figure 1). The digits $(5.0 \times 3.5 \mathrm{~cm})$ were surrounded by a rectangular frame measuring $7.5 \times 6.5 \mathrm{~cm}$, and constituting a visual angle of $5.3^{\circ}$ wide. Both the digit and the frame consisted of adjacent white dots ( $7 \mathrm{~mm}$ diameter, $3 \mathrm{~cd} / \mathrm{m}^{2}$ luminance) against a black background $\left(0.05 \mathrm{~cd} / \mathrm{m}^{2}\right.$ luminance $)$. In the intact form, the digit consisted of 14 dots and the frame of 36 dots. A degraded form was a deformation of the intact form, in which 10 dots from the frame were pseudorandomly distributed around the digit within the frame, thus securing a constant illumination of the stimulus that was independent of its form. The degraded digit remained unambiguously identifiable in that no conspicuous alternative digit emerged from a combination of the digit at issue and any dots of 


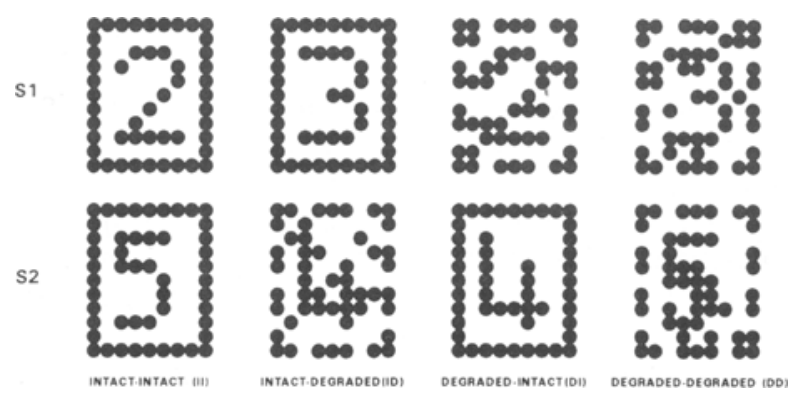

Figure 1. An overview of the visual stimulus conditions. The stimuli are depicted as negatives of the authentic stimuli. The stimuli presented first (S1) are depicted in the upper row, the subsequent presented stimuli (S2) in the bottom row. The stimulus conditions are constituted by the stimulus qualities of $S 1$ and $S 2$. The condition name is below each S1-S2 pair; abbreviations in parentheses are used throughout the text. Note that the digits and noise patterns represented by $S 1$ and $S 2$ are always different.

the noise pattern. There were four versions of each degraded digit, so as to prevent pattern-specific encoding. Noise patterns were also different between the digits.

Task. A trial of the experimental task started with the onset of a light-emitting diode (LED) underneath the left window for $1 \mathrm{sec}$, summoning the subject to fixate the center of that window. An interstimulus interval of $1.5 \mathrm{sec}$ separated the offset of the LED from the presentation of a visual stimulus (S1) on the left window. S1 was either an intact or a degraded digit, whose value indicated whether fixation on S1 should be continued ("no go") or a quick saccade should be made to the right window ("go"). In case of a go response, the presentation of S2 was triggered by the onset of the saccadic eye movement. As has been shown by Houtmans and Sanders (1983), subjects are unable to do any processing on S2 during the saccadic eye movements. S2 was also either an intact or a degraded digit, whose value determined the response key to be pressed. The offset of both stimuli took place $4 \mathrm{sec}$ after the onset of S1. The next trial started $4 \mathrm{sec}$ after the offset of the stimuli. A trial of the auditory control task was identical to a trial of the experimental task, except for one point. The stimulus that was presented while the subjects fixated the left window was not a digit, but a tone presented for $100 \mathrm{msec}$, whose pitch determined the go/ no-go saccadic response.

The assignment of digits and pitch to the responses requested by $\mathrm{S} 1$ and $\mathrm{S} 2$ was as follows. The total set of digits was subdivided into two sets: $\{2,3\}$ and $\{4,5\}$. For half of the subjects the set $\{2,3\}$ was used as $\mathrm{S} 1$ and $\{4,5\}$ as $\mathrm{S} 2$, whereas for the other half of the subjects this was reversed. Again, for half of the subjects of each group, the lower digit at $\mathrm{S} 1$ indicated the go response and the higher one the no-go response, whereas this was reversed for the other half of the subjects. At S2, the lower digit was always assigned to the left response key and the higher digit to the right one, since this proved to be the compatible stimulus-response setting. The assignment of pitch in the control task to the go and no-go response was compatible with the subject's digit condition in the experimental task, with a go response to the lower digit yoked to a go response to the low-pitched tone, and vice versa.

Design and Procedure. The independent combination of the visual qualities of $\$ 1$ and $\$ 2$ yielded four experimental conditions: intact-intact, intact-degraded, degraded-intact, and degradeddegraded. An example of each condition is depicted in Figure 1. In the control conditions, $\mathrm{S} 2$ was preceded by a tone, yielding toneintact and tone-degraded pairs. One group of subjects, the "blocked" group, received the total of six conditions in six separate blocks, while the other "mixed" group received intact and degraded S2s mixed within blocks. This left three separate blocks, determined by the quality of S1 (being intact, degraded, or a tone). Blocks were presented in a Latin-square arrangement in both groups. There were three dependent variables: (1) T1, the time between the presentation of $\mathrm{Sl}$ and the initiation of the saccade, (2) Tmov, the time between the initiation and the completion of the saccade, and (3) T2, the time between the completion of the saccade and pressing the response key.

The subjects were tested for $3 \mathrm{~h}$ in either in the morning or the afternoon. They were instructed not to move their heads during the execution of the task. Speed and accuracy of responses were equally stressed, both with respect to responding to $S 1$ and S2. All practice and experimental blocks contained 34 trials. Each trial had an equal probability of a go or no-go response at $\mathrm{S} 1$ and of a right or left response at $\mathbf{S} 2$ and, for the mixed group, an equal probability of an intact or a degraded S2. Furthermore, for the degraded digits, each noise pattern had an equal chance of occurrence, as had the specific combinations of noise patterns of S1-S2 pairs in the degraded-degraded condition. The subjects received one practice block in all experimental and control conditions, after which these conditions were presented for measurement. The blocked group received two series of six blocks in each $\mathrm{S} 1-\mathrm{S} 2$ condition, and the mixed group received three series of three blocks in each S1 condition. ${ }^{1}$ After a block, the subjects were given feedback on error performance and information about the stimulus condition of the next block. Breaks of about 5 min were inserted after training and after each series of blocks.

Data analysis. The on-line calculation of the initiation and completion times of saccades was carried out as follows. First, the point in time was established with the maximal tangent to the EOG sample values. Then, starting from this point, the first points back and forth in time of which the tangent approached zero were determined and regarded as the saccade initiation and completion times, respectively. Incorrect scorings due to eventual artifacts in the EOG signal were corrected afterward by the experimenter. This procedure yielded the points in time necessary for the calculation of T1, Tmov, and T2.

The resulting $\mathrm{T} 1$, Tmov, and $\mathrm{T} 2$ were pooled across series, after which means were calculated for each specific S1-S2 condition. The first two trials of each block and trials in which T1, Tmov, or T2 deviated more than two standard deviations from the mean were excluded from analysis. Error trials with respect to S1 (saccade initiation on a no-go trial) or $\mathbf{S} 2$ (pressing the wrong response key) were separately analyzed.

Separate univariate analyses of variance (ANOVAs) were carried out on mean T1, Tmov, and T2, as well as on mean error performance with respect to $S 1$ and $S 2$. These analyses were carried out on the data resulting from the experimental conditions only. $\mathrm{S} 1$ quality and $\mathbf{S} 2$ quality were within-subjects factors, whereas S2 presentation (blocked vs. mixed) and digit assignment $(\{2,3\} /\{4,5\}$ vs. $\{4,5\} /\{2,3\})$ were between-subjects factors. An additional analysis was carried out with respect to $T 2$, in which the results from the main experimental conditions were contrasted with those from the auditory conditions.

\section{Results}

In this section, the results regarding $\mathrm{T} 2$ are presented first, since they are of main interest for the present study. Then the results regarding $\mathrm{T} 1$ and Tmov are presented, followed by the data on error performance. Furthermore, to allow an easy reference to specific stimulus conditions, the following abbreviations are used throughout this section: I and D for intact and degraded digits, respectively, and $A$ for the auditory signal. Pairs of these abbreviations denote the specific stimulus conditions. For instance, $\mathrm{DI}$ indicates that $\mathrm{S} 1$ was a degraded digit and $\mathrm{S} 2$ was an 

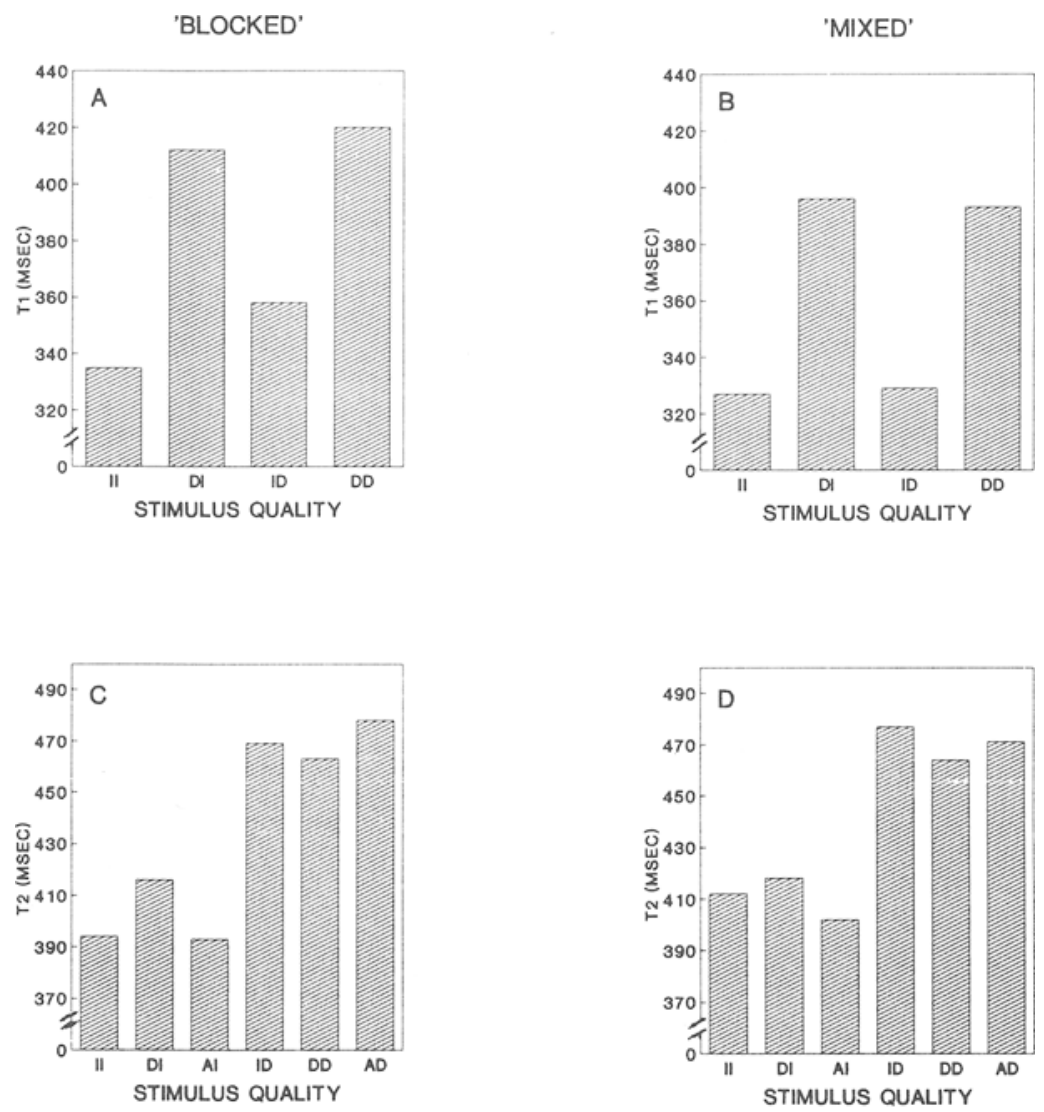

\begin{abstract}
Figure 2. Mean response times in Experiment 1 as a function of $\mathrm{S} 1$ quality, $\mathrm{S} 2$ quality, and S2 presentation (blocked, panels $A$ and C; mixed, panels B and D). T1 is the response time to $S 1$, and $T 2$ is the response time to $S 2 . I=$ intact stimuli; $D=$ degraded stimuli; $\mathbf{A}=$ auditory stimuli.
\end{abstract}

intact digit, $\mathrm{AD}$ indicates that $\mathrm{S} 1$ was a tone and $\mathrm{S} 2$ was a degraded digit, and so on.

Figures $2 \mathrm{C}$ and $2 \mathrm{D}$ show the mean $\mathrm{T} 2$ as a function of S1 and S2 quality for the blocked and mixed groups, respectively. The ANOVA revealed a main effect of S2 quality $\left[F(1,20)=242.24, M S_{\mathrm{e}}=338.58, p<.001\right]$, indicating that responding to an intact $\mathrm{S} 2$ was faster than to a degraded S2. This effect interacted with S1 quality $\left[F(1,20)=10.87, M S_{\mathrm{e}}=304.81, p<.01\right]$, reflecting the HSE. There was no indication that the HSE depended on the presentation of intact and degraded S2s in pure or mixed blocks of trials, as testified by an absence of the S1 quality $\times$ S2 quality $\times$ S2 presentation interaction $\left[F(1,20)=0.33, M S_{\mathrm{e}}=304.81, p=.57\right]$. Planned comparisons revealed that the response to an intact $\mathrm{S} 2$ was faster when it followed an intact S1 than when it followed a degraded S1; that is, T2(II) < T2(DI) $[F(1,20)=12.64$, $\left.M S_{\mathrm{e}}=367.65, p<.01\right]$. The corresponding tendency to respond faster to a degraded S2 when preceded by a degraded $\mathrm{S} 1$ than when preceded by an intact $\mathrm{S} 1$ was not significant $\left[F(1,20)=2.18, M S_{\mathrm{e}}=1,012.25, p=.16\right]$. Finally, some significant effects related to the grouping factor "digit assignment" were observed, but since these effects did not seem to affect the conclusions with respect to the HSE, they are ignored here and in the following experiments.

An additional analysis contrasted the HSE on T2 with the auditory control conditions, in which a tone preceded the intact or degraded $S 2$. This analysis revealed that the $\mathrm{AD}-\mathrm{AI}$ difference did not significantly differ from the IDII difference $\left[F(1,20)=0.95, M S_{\mathrm{e}}=1,191, p=.34\right]$, whereas the AD-AI difference was significantly larger than the DD-DI difference $\left[F(1,20)=14.61, M S_{\mathrm{e}}=\right.$ $1,515.26, p<.001]$. Thus, compared with the auditory conditions, the present HSE is a manifestation of deviant behavior following a degraded S1. This was specified in greater detail by simple contrasts, which revealed that the T2s of the S1-auditory and the S1-intact conditions were also absolutely comparable; that is, neither $\mathrm{T} 2(\mathrm{AI})$ and T2(II) $\left[F(1,20)=1.37, M S_{\mathrm{e}}=589.64, p=.26\right]$ nor $\mathrm{T} 2(\mathrm{AD})$ and T2(ID) $\left[F(1,20)=0.02, M S_{\mathrm{e}}=1,241.60\right.$, $p=.88\}$ differed significantly. By contrast, T2(DI) was significantly longer than $\mathrm{T} 2(\mathrm{AI})\left[F(1,20)=37.56, M S_{\mathrm{e}}=\right.$ $248.18, p<.001$ ], whereas T2(DD) tended to be some- 
what shorter than $\mathrm{T} 2(\mathrm{AD})\left[F(1,20)=1.82, M S_{\mathrm{e}}=1,501\right.$, $p=.19]$.

Figures $2 \mathrm{~A}$ and $2 \mathrm{~B}$ show the mean $\mathrm{T} 1$ from the experimental conditions as a function of $\mathrm{S} 1$ and $\mathrm{S} 2$ quality. The ANOVA revealed a significant main effect of $\mathrm{S} 1$ quality $\left[F(1,20)=65.91, M S_{\mathrm{e}}=1,677.54, p<.001\right]$, indicating a saccade initiation that was faster following an intact S1 than following a degraded S1. Furthermore, an interaction between the effects of S2 quality and S2 presentation $\left[F(1,20)=5.12, M S_{\mathrm{e}}=309.20, p<.05\right]$ indicates that, in the blocked group, the saccade was initiated faster when an intact S2 followed S1 than when a degraded $\mathrm{S} 2$ followed $\mathrm{S} 1$, whereas this difference was absent in the mixed group.

The average Tmov amounted to $138 \mathrm{msec}$. Tmov was significantly affected by S2 quality $[F(1,20)=7.01$, $\left.M S_{\mathrm{e}}=3.15, p<.05\right]$, although the saccade preceding an intact $\mathrm{S} 2$ was only $1 \mathrm{msec}$ faster than the saccade preceding a degraded $\mathbf{S} 2$. This effect occurred only in the blocked group (a 2-msec effect), and not in the mixed group, as indicated by an interaction between the effects of S2 quality and S2 presentation $\left[F(1,20)=7.63, M S_{\mathrm{e}}=\right.$ $3.15, p<.05]$. Generally, differences on Tmov are readily significant due to a very high consistency among subjects, but they typically do not exceed $2 \mathrm{msec}$ and thus are not considered to be of interest for the present study.

Table 1 shows the error percentages that occurred in the experimental and control conditions of the blocked and mixed groups. With respect to Sl, the ANOVA did not reveal any significant differences between conditions. With respect to $\mathrm{S} 2$, the ANOVA revealed a main effect of $\mathrm{S} 2$ quality $\left[F(1,20)=8.26, M S_{\mathrm{e}}=12.09, p<.01\right]$, indicating that fewer errors were made when S2 was intact than when S2 was degraded ( $1.75 \%$ vs. $3.78 \%$, respectively). This effect depended on the quality of $\mathrm{S} 1[F(1,20)$ $=7.58, M S_{e}=4.35, p<.05$ ], constituting an HSE on error performance, which in turn depended on $\mathrm{S} 2$ presentation $\left[F(1,20)=4.58, M S_{\mathrm{e}}=4.35, p<.05\right]$. The latter three-way interaction indicates that the HSE on error performance was present in the mixed group, in that fewer errors occurred in the homogeneous conditions than in

Table 1

Mean Error Percentages in Responding to S1 and S2 in the Experimental and Control Conditions of Experiments 1 and 2

\begin{tabular}{|c|c|c|c|c|c|c|}
\hline & \multicolumn{4}{|c|}{ Experimental } & \multicolumn{2}{|c|}{ Control } \\
\hline & II & DI & ID & $\mathrm{DD}$ & AI & $\mathrm{AD}$ \\
\hline \multicolumn{7}{|c|}{ Experiment 1: Blocked } \\
\hline S1 & 3.39 & 3.65 & 3.13 & 4.95 & 6.77 & 7.29 \\
\hline S2 & 2.08 & 2.08 & 3.65 & 3.13 & 2.08 & 3.91 \\
\hline \multicolumn{7}{|c|}{ Experiment 1: Mixed } \\
\hline S1 & 3.30 & 2.60 & 3.30 & 2.60 & 2.95 & 2.95 \\
\hline S2 & 0.35 & 2.43 & 5.21 & 3.13 & 2.08 & 3.13 \\
\hline \multicolumn{7}{|c|}{ Experiment 2} \\
\hline SI & 3.39 & 1.56 & 1.56 & 3.65 & 4.69 & 5.99 \\
\hline$\$ 2$ & 2.08 & 1.04 & 2.87 & 3.39 & 3.13 & 4.69 \\
\hline
\end{tabular}

Note $-\mathrm{I}=$ intact stimuli; $\mathrm{D}=$ degraded stimuli; $\mathrm{A}=$ auditory stimuli. the heterogeneous conditions ( $1.74 \%$ vs. $3.82 \%$, respectively), but not in the blocked group ( $2.61 \%$ vs. $2.87 \%$, respectively). Finally, simple contrasts of the experimental conditions with auditory conditions did not yield any significant differences. In sum, the data on error performance show that the HSE, as found on T2, was not an artifact due to differences in speed-accuracy tradeoff between conditions, because the "faster" conditions tended to produce fewer errors.

\section{Discussion}

The main result of Experiment 1 was the replication of Hansen and Sanders's (1988) HSE. This effect indicates that T2 was shorter when S2 had the same quality as the preceding $\mathbf{S} 1$ than when $\mathrm{S} 2$ had a different quality from the preceding S1. In the present study, matching was excluded as an explanation for this effect, because $\mathrm{S} 1$ and S2 required independent responses and aiways represented different digits and noise patterns. Thus, an explanation should proceed from the specific processing demands of intact and degraded stimuli.

Apart from this main result, two other results are of importance. First, by mixing intact and degraded S2s, the HSE was divided across T2 and error distributions rather than reduced. A reduction was expected if the HSE relied on a preparatory mechanism to deal with the worst case expected on a trial, because absence of knowledge on the forthcoming S2 provokes the selection of the slow processing route in all stimulus conditions. Effects that are possibly related to this worst case principle occurred, in fact, in the blocked group, where saccadic eye movements were initiated and executed faster when in anticipation of an intact S2 than when in anticipation of a degraded S2. However, the data of the mixed group show that these effects can be dissociated from the HSE on T2. Thus, although advance knowledge on the forthcoming S2 had several strategic effects on prior events, it did not cause the HSE on T2. The second important result is that, compared with the auditory conditions, the present HSE manifested itself neither as a facilitating effect nor as an inhibiting effect. Instead, the auditory conditions mimicked the results of the S1-intact conditions with respect to T2. The implications of this finding will be further elaborated in the General Discussion.

\section{EXPERIMENT 2}

The HSE in Experiment 1 was quite small. Because of this, it was difficult to acquire insight into the effect size of its various components relative to each other and relative to the auditory conditions. Experiment 2, then, was an attempt to magnify the HSE by increasing the processing demands of degraded stimuli. For this purpose, degraded stimuli were presented $180^{\circ}$ rotated, so as to increase the difference in processing demands between intact and degraded stimuli. This manipulation added some $80 \mathrm{msec}$ to the reaction time to degraded, upright stimuli in a study by Sanders and Rath (1991), who used the digits 
2-7 for S1 and S2 in a matching task. An interesting result of their study was that T2 to an intact S2 was significantly shorter when S1 was degraded than when S1 was both degraded and rotated. Referring to Hansen and Sanders (1988), Sanders and Rath attributed this effect to differences in matching times, but a procedural account again poses a viable alternative.

\section{Method}

The method was the same as that used for the blocked condition of Experiment 1, except for two points. First, there was a change in stimulus material with respect to the degraded stimuli, which were presented $180^{\circ}$ rotated, yielding degraded and rotated stimuli. Intact digits, by contrast, were presented in their normal, upright position. Second, because problems were expected in distinguishing " 2 " and " 3 " in the degraded category, the digit sets were changed to $\{2,4\}$ for $S 1$, and $\{3,5\}$ for $S 2$, or vice versa. $S 1$ and S2 quality were the within-subjects factors in the ANOVAs, and digit assignment $(\{2,4\} /\{3,5\}$ vs. $\{3,5\} /\{2,4\})$ and session (the first or second series of blocks) were the between-subjects factors.

Subjects. Six male and 6 female students, none of whom had engaged in the previous experiment, served as paid subjects. Two of the subjects were unable to recognize several of the degraded digits after practice, and therefore were replaced by 2 others.

\section{Results}

Figure 3B shows the mean $\mathrm{T} 2$ as a function of $\mathrm{S} 1$ and S2 quality. The effect of S2 quality was highly significant $\left[F(1,10)=372.04, M S_{\mathrm{e}}=807.41, p<.001\right]$ and interacted with the effect of S1 quality $[F(1,10)=24.40$, $\left.M S_{\mathrm{e}}=803.77, p<.001\right]$. This interaction reflects the HSE that was also found in Experiment 1. Planned comparisons revealed that $\mathrm{T} 2$ was shorter when the stimuli were homogeneous than when they were not. That is, a shorter T2 was found at II than at DI $[F(1,10)=27.54$, $\left.M S_{\mathrm{e}}=1,581.95, p<.001\right]$, and a shorter $\mathrm{T} 2$ at $\mathrm{DD}$ than at ID $\left[F(1,10)=6.65, M S_{\mathrm{e}}=5,277.48, p<.05\right]$. Planned comparisons to the auditory conditions replicated the main result of Experiment 1, in that the effects of the auditory conditions and the $\mathrm{S} 1$-intact conditions were additive [AD-AI $=\mathrm{ID}-\mathrm{II}, F(1,10)=0.60, M S_{\mathrm{e}}=11,248.95$, $p=.46]$, whereas the effects of the auditory conditions and the S1-degraded conditions interacted [AD-AI > DD-DI, $\left.F(1,10)=26.49, M S_{\mathrm{e}}=3,716.88, p<.001\right]$. Furthermore, simple contrasts between the components of the HSE and auditory conditions also yielded results that were very similar to those obtained in Experiment 1 . That is, $\mathrm{T} 2$ at $\mathrm{AI}$ was not different from $\mathrm{T} 2$ at II $[F(1,10)=$ $\left.0.39, M S_{\mathrm{e}}=1,302.47, p=.55\right]$, and $\mathrm{T} 2$ at $\mathrm{AD}$ was not different from $\mathrm{T} 2$ at $\operatorname{ID}\left[F(1,10)=0.47, M S_{\mathrm{e}}=7,571.62\right.$, $p=.51]$. By contrast, $\mathrm{T} 2$ at $\mathrm{DI}$ was longer than at $\mathrm{AI}$ $\left[F(1,10)=17.84, M S_{\mathrm{e}}=1,943.02, p<.01\right]$, whereas T2 at DD was shorter than at $\mathrm{AD}\left[F(1,10)=5.39, M S_{\mathrm{e}}\right.$ $=3,023.27, p<.05]$.

Figure $3 \mathrm{~A}$ shows $\mathrm{T} 1$ resulting from the experimental conditions as a function of $S 1$ and $S 2$ quality. A main effect of $S 1$ quality $\left[F(1,10)=41.39, M S_{\mathrm{e}}=3,500.04\right.$, $p<.001]$ reflected faster responding to intact stimuli than to degraded stimuli. Most importantly however, in contrast with the blocked condition of Experiment 1, there was neither a main effect of S2 quality $[F(1,10)=0.00$, $\left.M S_{\mathrm{e}}=1,199.94\right]$, nor an interaction between the effects of $\mathrm{S} 1$ and $\mathrm{S} 2$ quality $\left[F(1,10)=0.23, M S_{\mathrm{e}}=499.75\right]$. Also, there were some significant effects involving the factors of session and digit assignment. However, these variables did not affect the findings that are of importance to the present study, so their effects will be ignored. Finally, Tmov $(M=139 \mathrm{msec})$ did not vary significantly among conditions.

Error percentages (Table 1) remained well below 5\%. Slightly more errors occurred in the homogeneous than in the heterogeneous stimulus conditions, but the ANOVAs on error performance with respect to S1 and S2 did not yield any significant difference between these means, and the simple contrast between the experimental and auditory conditions did not result in significance.

\section{Discussion}

Experiment 2 proved to be successful as an attempt to magnify the HSE by increasing the differences in processing demands between intact and degraded stimuli. The sizable HSE on T2 again indicated that the response to
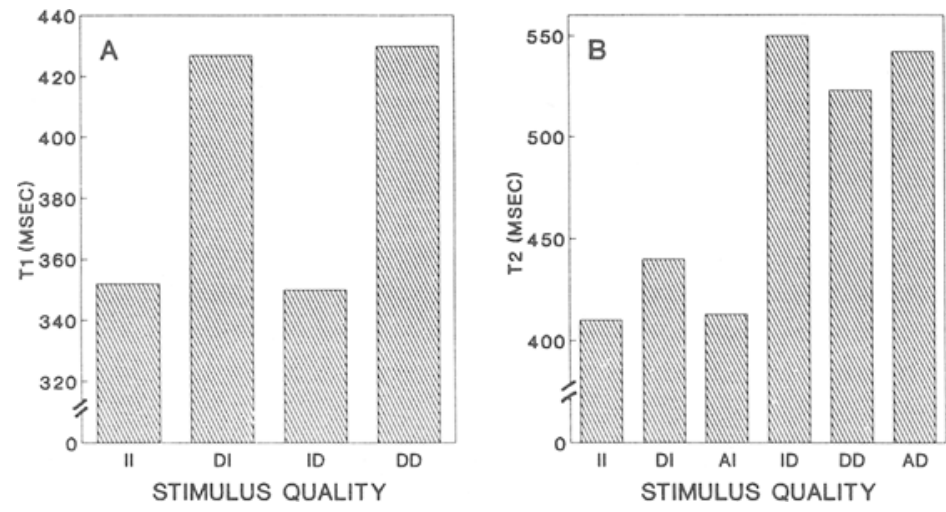

Figure 3. Mean response times in Experiment 2 as a function of $S 1$ and $S 2$ quality. $T 1$ is the response time to $S 1$, and $T 2$ is the response time to $S 2 . I=$ intact stimuli; $\mathbf{D}=$ degraded stimuli; $\mathbf{A}=$ auditory stimuli. 
S2 was faster when stimulus pairs were homogeneous than when they were not. This result replicated the main result of Experiment 1. Another excellent replication concerned the observation that a tone and an intact $\mathrm{S} 1$ yielded identical results with respect to $T 2$. Thus, relative to the auditory conditions, it was again a degraded $\mathrm{S} 1$ that somehow caused deviant behavior on T2. Furthermore, the degraded manipulation proved to be strong enough to demonstrate significant differences between all separate homogeneous and heterogeneous components, as well as between the components of the auditory and S1-degraded conditions. One result deviating from Experiment 1 was that, although the stimulus conditions were presented in pure blocks of trials and the subjects had advance knowledge of the qualities of the forthcoming S1 and S2, no effects of S2 quality were observed on either T1 or Tmov. Thus, in line with the data of the mixed condition of Experiment 1, Experiment 2 provided another case in which dissociation occurred between strategic effects on $\mathrm{T} 1$ and Tmov on the one hand and the HSE on T2 on the other, although it is not clear what exactly caused this deviant result from the blocked condition of Experiment 1. In all, Experiment 2 provided a useful replication of the data of Experiment 1 in that it confirmed the main findings, while it shed more light on the relations of the separate components of the HSE relative to each other and relative to the auditory conditions.

\section{EXPERIMENT 3}

The primary aim of the experiments conducted so far has been to demonstrate that the HSE is not dependent on the presence or activation of representations. This was achieved by restricting the overlap between $\mathrm{S} 1$ and $\mathrm{S} 2$ to the level of stimulus quality, implying that the digits and the specific noise patterns of S1 and S2 were always different. Experiment 3 was an examination of whether sameness of digits and noise patterns would further add to the HSE, as was the case in Hansen and Sanders's (1988) study. For this purpose, nominally same and different digits were used for the S1-S2 pairs, constituting two levels of the variable digit correspondence. The different condition was exclusively used in the previous experiments and served in Experiment 3 as a baseline for the evaluation of the effects resulting from the same condition.

As far as the stimulus conditions are concerned, Experiment 3 comes very close to Hansen and Sanders's (1988) experiment described in the introduction, but the present task is different in that matching is not required, because $S 1$ and $S 2$ require independent responses. Thus, following Proctor's (1981) argument, if the main findings of Hansen and Sanders can be replicated, a procedural account should be favored over a representational one.

\section{Method}

Relative to the preceding experiments, the following adjustments were made. Regarding S1, the digit set $\{2,3,5,6,8,9\}$ was used, with the subset $\{2,3,5,6\}$ requiring a go response and the subset $\{8,9\}$ requiring a no-go response, for all the subjects. Regarding
S2, the digit set was identical to the set requiring a go response at $\mathrm{S} 1$, that is, the set $\{2,3,5,6\}$. Of this set, the subset $\{2,3\}$ required a left manual response and the subset $\{5,6\}$ required a right manual response. Note that by leaving out the digits 4 and 7 , isolated groups of digits were created, so that the decision processes that they give rise to would be facilitated.

In addition to the variables of stimulus quality employed in the previous experiments, digit correspondence was added as an independent variable, comprising a same and a different condition. In the same condition, the digits of S1 and $\mathrm{S} 2$ were identical, whereas the digits were different in the different condition. Thus, the same condition contained the $S 1-S 2$ pairs 2-2,3-3,5-5, and 6-6 in all conditions of stimulus quality, whereas the different condition contained all the other possible pairs.

Each experimental block contained 50 trials, of which the first 2 were discarded. The 48 remaining trials were composed such that all individual stimuli had an equal probability of presentation, with one exception. Given that in the degraded-degraded condition S1 and $S 2$ were same (an event with a probability of .25 ), the probability that their noise patterns were identical was .5 instead of .25 , which would have been the expected probability because four different noise patterns were used for each digit. The reason for this deviation was to obtain equal numbers of trials in which same stimuli had identical noise patterns and in which they had different noise patterns, so as to enable a fair comparison between these specific same conditions. Thus, each block contained a random presentation of $(1 / 3 \times 48) 16$ no-go trials and $(2 / 3 \times 48) 32$ go trials. The go trials comprised $(3 / 4 \times 32) 24$ different trials and $(1 / 4 \times 32) 8$ same trials. In the condition with two degraded stimuli $(1 / 2 \times 8)$, four same digits had identical noise patterns, whereas the other $(1 / 2$ $\times 8$ ) four same stimuli had different noise patterns.

Auditory conditions were omitted, because the previous experiments demonstrated that conditions with an intact S1 yielded identical results, as far as $\mathrm{T} 2$ is concerned. Thus, there were eight experimental conditions, constituted by an independent combination of S1 quality (2), S2 quality (2), and digit correspondence (2). The visual qualities of S1 and S2 were varied between blocks, as in the blocked condition of Experiment 1, whereas digit correspondence naturally occurred within blocks. Four separate blocks, constituted by the quality of $\mathrm{S} 1$ and $\mathrm{S} 2$, were presented four times each in a Latin-square arrangement. Means of the pooled data of each stimulus condition were calculated and subsequently entered into ANOVAs with S1 quality, S2 quality, and digit correspondence (same/different) as independent factors.

Subjects. Twelve new subjects ( 6 females $/ 6$ males) were tested during about $4 \mathrm{~h}$ and paid for their services. One subject was replaced by another, because of unacceptable error rates.

\section{Results}

Although in the DD condition same pairs with identical noise patterns had a shorter T2 than same pairs with different noise patterns (496 vs. $512 \mathrm{msec}$ ), a preliminary $t$ test showed that this difference was not significant $[t(11)=1.41, p=.28]$, due to a strong variability between subjects. For this reason, the data of these conditions were collapsed and treated as a single same factor.

Figures $4 \mathrm{~B}$ and $4 \mathrm{C}$ show the mean $\mathrm{T} 2$ as a function of S1 and S2 quality for different and same stimuli, respectively. The ANOVA revealed a significant main effect of S2 quality $\left[F(1,11)=65.89, M S_{\mathrm{e}}=1,216.51, p<.001\right]$, which interacted with the effect of $S 1$ quality $[F(1,11)=$ $\left.12.93, M S_{\mathrm{e}}=450.66, p<.01\right]$, reflecting the HSE. The HSE depended, in turn, on digit correspondence $[F(1,11)$ $\left.=7.29, M S_{\mathrm{e}}=208.44, p<.05\right]$. This effect reflects a more robust HSE in the case of same stimuli, although 

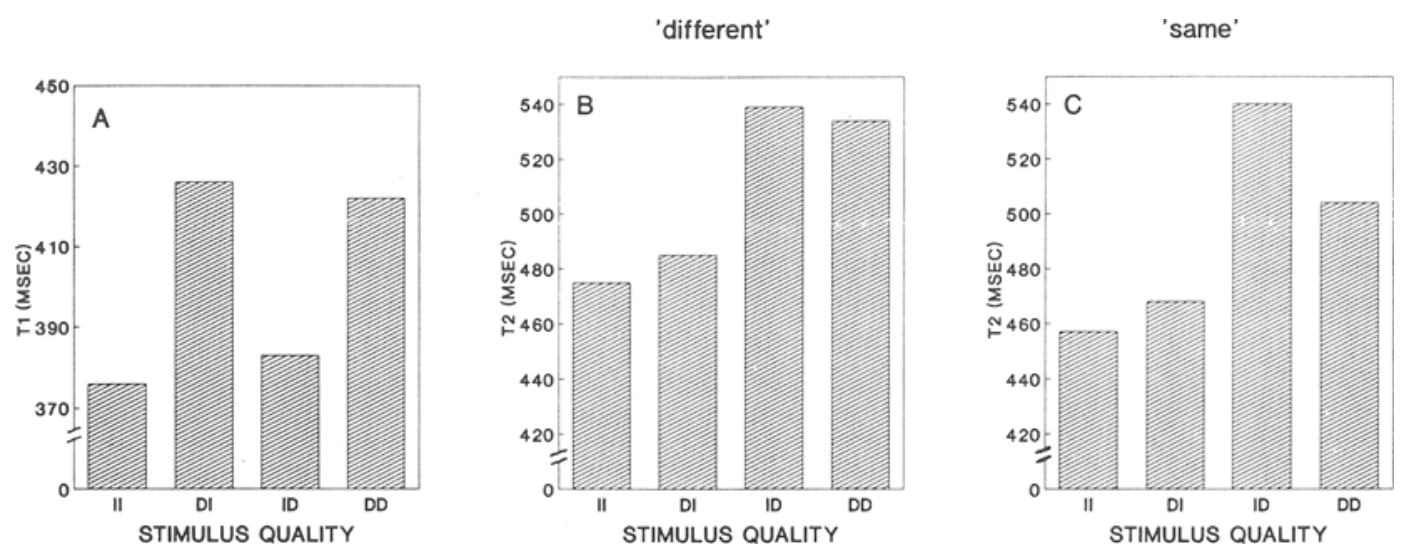

Figure 4. Mean response times in Experiment 3 as a function of S1 quality, S2 quality, and digit correspondence (same or different). T1 is the response time to S1, and T2 is the response time to S2. I = intact stimuli; D = degraded stimuli.

a planned comparison showed that the HSE was still present in the case of different stimuli $[F(1,11)=4.87$, $\left.M S_{\mathrm{e}}=573.11, p=.05\right]$, corroborating the results of the previous two experiments. In further agreement with Experiment 1, comparisons between the components of the HSE in the different condition yielded a longer T2 at DI than at II $\left[F(1,11)=6.85, M S_{\mathrm{e}}=172.27, p<.05\right]$, while T2 at DD was not significantly shorter than at ID $\left[F(1,11)=1.27, M S_{\mathrm{e}}=268.42, p=.28\right]$. This pattern was reversed on same trials, in which responding at DI was not significantly slower than at II $[F(1,11)=1.68$, $M S_{\mathrm{e}}=938.44, p=.23 \mathrm{~J}$, but responding at DD was faster than at ID $\left[F(1,11)=13.71, M S_{\mathrm{e}}=1,113.24, p<.01\right]$. The ANOVA showed, furthermore, that the faster response to same stimuli than to different stimuli was no more than a trend $\left[F(1,11)=3.48, M S_{\mathrm{e}}=1,692.33, p=.09\right]$. Planned contrasts showed that this was mainly due to the absence of any effect of digit correspondence at ID $[F(1,11)=$ $0.01, M S_{\mathrm{e}}=2,099.36$, which was, interestingly, also the case in the study by Hansen and Sanders (1988). In all other conditions of stimulus quality, there was at least a trend toward a faster response to same stimuli than to different stimuli at II $\left[F(1,11)=3.97, M S_{\mathrm{e}}=978.36\right.$, $p=.07]$, DI $\left[F(1,11)=4.00, M S_{\mathrm{e}}=815.91, p=.07\right]$, and $\mathrm{DD}\left[F(1,11)=9.67, M S_{\mathrm{e}}=1,062.21, p<.01\right]$.

Figure 4A shows the mean $\mathrm{T} 1$ as a function of $\mathrm{S} 1$ and S2 quality. The only significant effect revealed by the ANOVA was the main effect of $S 1$ quality $[F(1,11)=$ $\left.115.47, M S_{\mathrm{e}}=409.68, p<.001\right]$, reflecting faster responding to intact than to degraded digits. The effect suggested by Figure 4, that $S 1$ of homogeneous stimulus pairs (II and DD) was responded to faster than S1 of heterogeneous stimulus pairs (ID and DI, respectively), failed to reach significance $\left[F(1,11)=3.77, M S_{\mathrm{e}}=191.11, p=.08\right]$.

The mean Tmov amounted to $141 \mathrm{msec}$ and again showed maximal deviations of $2 \mathrm{msec}$ among conditions. As in the blocked condition of Experiment 1, the saccade duration was slightly but significantly shorter $(1 \mathrm{msec})$ when the subjects anticipated an intact $\mathrm{S} 2$ rather than a degraded $\mathrm{S} 2\left[F(1,11)=5.69, M S_{\mathrm{e}}=5.64, p<.05\right]$.

Error percentages are presented in Table 2. The ANOVA did not reveal any significant differences in error performance among conditions for either S1 or S2.

\section{Discussion}

Three main results of Experiment 3 are better explained in procedural terms than in terms of matching representations. First, in agreement with the previous two experiments, it was found that a small but significant HSE on $\mathrm{T} 2$ was brought about by digits of a different value. As argued previously, the physical dissimilarity of these stimuli and the absence of matching requirements exclude a representational account for this effect. Second, there was a tendency for $\mathrm{T} 2$ to be shorter for same stimuli than for different stimuli, which is in agreement with Proctor's (1981) original finding that the matching of stimuli is not a requirement to observe the fast-same effect. Although same representations may have a stronger physical resemblance than different representations, a procedural account in terms of encoding facilitation is still more obvious than a matching account, because of the inadequacy of matching to arrive at the correct response in the present task. Finally, in agreement with the result found by Hansen and Sanders (1988) in a matching task, same stimuli yielded a more robust HSE than different stimuli. This interaction suggests that the fast-same effect and the HSE have a com-

Table 2

Mean Error Percentages in Responding to S1 and S2 in Experiment 3

\begin{tabular}{lcccc}
\hline & \multicolumn{4}{c}{ Stimulus Condition } \\
\cline { 2 - 5 } & II & DI & ID & DD \\
\hline S1 & 5.99 & 5.73 & 4.82 & 5.99 \\
S2 different & 3.04 & 3.56 & 3.47 & 2.95 \\
S2 same & 3.39 & 3.65 & 3.65 & 4.17 \\
\hline
\end{tabular}

Note-I $=$ intact stimuli; $D=$ degraded stimuli. 
mon underlying source, which completes a coherent picture outlined by the first two main results. In sum, then, because of the absence of matching requirements in the present task, the HSE and the fast-same effect, as well as their interaction, are probably not caused by any mechanism based on representations, but rather by a mechanism based on encoding procedures.

A puzzling result of Experiment 3 concerns the absence of any facilitation on T2 of same stimuli relative to different stimuli in the condition with an intact $\mathrm{Sl}$ and a degraded S2, while this facilitation was, at least as a trend, present in other conditions. This seems to be more than a coincidence, because the same result was found by Hansen and Sanders (1988) in their matching task. It is important to note that this absence of a fast-same effect cannot be explained as a lack of correspondence between same representations of intact and degraded stimuli, because a strong trend toward a fast-same effect was observed both in a degraded S1 and an intact S2, and in two degraded digits with different noise patterns. However, a harmonious procedural account for this result is also not readily apparent.

\section{GENERAL DISCUSSION}

All three experiments showed the HSE, as previously found by Hansen and Sanders (1988): The response following the second stimulus of a pair is faster when the stimuli share the same structural properties than when they do not. The rationale of the design of the present study was to restrict the overlap of actions with respect to $S 1$ and S2 to encoding processes, while excluding the identity of digits and noise patterns. Thus, it is concluded that the occurrence of the HSE is not dependent on a process of matching S1 and S2 (Experiments 1-3), or on any mechanism based on the identity of the representations of S1 and S2 (Experiments 1-2; Experiment 3, the different condition). Rather, the mere requirement of encoding S1 and $\mathbf{S} 2$ is a sufficient condition for the observation of the effect.

It could be argued that the present HSE was not symmetrical, in that the faster response to S2 at intact-intact pairs than to $\mathbf{S} 2$ at degraded-intact pairs did not always have a significant counterpart at degraded-degraded pairs relative to intact-degraded pairs (Experiments 1 and 3). It is, however, not clear how much attention this asymmetry deserves. The observed differences on $\mathrm{T} 2$ between degraded-degraded pairs and intact-degraded pairs in Experiments 1 and 3 seem too sizable to accept the null hypothesis of no effect. Furthermore, it could be that an additional hidden factor that broke the symmetry of the HSE was present. Suppose, for instance, that the task load varies with the number of degraded digits presented on a trial and, furthermore, that an increasing task load slows down the response to S2. It follows that $\mathrm{T} 2$ in the degraded-degraded condition is affected in opposite directions by two variables - task load and repetition of processing route-and so the advantage of repeating the slow processing route should be assessed as stronger than the present data suggest. ${ }^{2}$ Whatever the precise mechanism, it is not detrimental to the main conclusion that the interaction of the effects of S1 and S2 quality establishes the HSE as a procedural phenomenon.

In the remainder of this section, three more issues are addressed: the implications of the present findings for matching phenomena, the strategic contribution to the HSE, and the neutrality of the auditory conditions and its implications for the nature of the effect.

To obtain access to the first issue, a comparison is made between the present data and those of Hansen and Sanders (1988), who used stimulus conditions identical to those of the present study in a matching task. The comparison yields a clear analogy of the HSEs of both studies. In neither study was nominal sameness of the digits represented by $\mathrm{S} 1$ and $\mathrm{S} 2$ a prerequisite for the occurrence of the HSE, though it increased the magnitude of the HSE as compared with different stimuli. Furthermore, in both studies, the greater robustness of the HSE for nominal same stimuli was especially due to the absence of a fastsame effect in the intact-degraded condition. It should be admitted, though, that the present HSE remained quantitatively less than the HSE of Hansen and Sanders. Although the importance of this difference is not clear, because it did not proceed from a single experiment, caution in interpreting the similarities of both HSEs is in order. It is therefore not warranted to conclude that Hansen and Sanders's HSE relies entirely on the same procedural mechanism proposed for the present HSE. However, it is tempting to suppose that any larger effect in a matching task should be additive to the effect observed at present. If it is assumed that the matching process subsequently follows the identification of $\mathbf{S} 2$, variables found to affect $\mathrm{T} 2$ in an identification task should necessarily affect $T 2$ in a matching task in an identical way, although the latter task allows additional effects to occur during a possible process of matching representations of S1 and S2. Several models on matching phenomena, usually referred to as single-process models (Farell, 1985), in fact assume the seriality of identification and matching processes (e.g., Krueger, 1978; Proctor, 1981), and the observed analogy of the present HSE and that of Hansen and Sanders lends further support for this assumption.

The second issue readdresses the question of whether the HSE relies on a data-driven or a strategic mechanism. The first mechanism explains the HSE as the mere consequence of engaging in similar or dissimilar processing behavior before an actual stimulus is processed. By contrast, strategic accounts assume that advance preparation is the key to the occurrence of the effect. One possible strategic mechanism, based on the preparation for the worst case occurring on a trial, could not be confirmed by the data presently obtained, as already explained in the discussions to Experiments 1 and 2. Preparing for the worst case is, however, only one out of several possible strategic mechanisms that could explain the HSE, and so its disconfirmation does not necessarily imply the correct- 
ness of the alternative data-driven account. In particular, it could be that subjects always prepare for the quality of the first signal to come, and that it is this preparation that causes the subsequent HSE. To test this mechanism, subjects should be deprived of their advance knowledge on the quality of $\mathrm{S} 1$, by mixing its intact and degraded forms. In that case, stimulus-specific preparation regarding S1 would fail, and so the HSE should disappear if it relies on the strategic mechanism proposed here, but not if it relies on a data-driven mechanism.

The third issue to be discussed is whether the auditory conditions could be considered as neutral, and if so, what their neutrality implies for the nature of the HSE. As argued in the introduction, there are two challenges to the neutrality of the auditory conditions. First, an auditory S1 could provide a T2 that is shorter than it should be in an ideal neutral condition, due to its alerting property. Second, an auditory $\mathrm{S} 1$ could provide a $\mathrm{T} 2$ that is longer than it should be in an ideal neutral condition, due to refractoriness to switch from an auditory mode of processing to a visual one. From a theoretical point of view, only the second of these challenges should be seriously considered, because a great deal of evidence argues against the first one. An alerting effect of an auditory stimulus on the response to a visual command stimulus (i.e., S2 in the present study) is only observed under specific conditions, none of which are met in the present study. Specifically, (1) the auditory stimulus should have an intensity beyond $70 \mathrm{dBA},(2)$ the preparedness for processing the visual stimulus should be low, and (3) the relation of the visual stimulus to the response should be either simple or highly compatible (Sanders, 1980; Van der Molen \& Keuss, 1981). One finding argues, however, against both challenges to the neutrality of the auditory conditions, namely, the robust finding of Experiments 1 and 2 that, for $\mathrm{T} 2$, the auditory conditions yield results that are identical to the S1-intact conditions. Rejecting the neutrality of the auditory conditions implies the acceptance that the equality of $\mathrm{T} 2$ in these conditions, relative to the S1-intact conditions, relies on a coincidence. That is, facilitation or inhibition on $\mathrm{T} 2$ due to an auditory $\mathrm{S} 1$, as implied by the first and second challenge, respectively, is precisely offset by an equal amount of facilitation or inhibition in switching from processing an intact $\mathbf{S} 1$ to a visual S2. Although the possibility that this is what happens cannot be excluded, the more likely possibility is that the auditory conditions are indeed neutral.

The acceptance of the auditory conditions as neutral has an important implication for the nature of the HSE, namely, that this effect is caused by deviant behavior following the processing of a degraded S1. Processing a degraded $S 1$ delays the response to an intact $\mathrm{S} 2$ and tends to speed up the response to a degraded S2, whereas processing an intact $\mathrm{S} 1$ does not affect the responding to $\mathrm{S} 2$. In terms of the "alternative routes" model of Van Duren and Sanders (1988), this implies that switching from the slow route to the fast route is offset by costs, while a repetition of this route may yield some benefits. By contrast, using the fast route leaves subsequent processing unaffected, in that there are no costs involved in switching to the slow route or benefits in repeating the fast route. It seems, then, that using the slow route has the property of imposing an inflexibility on the processing system: it stimulates its reuse while blocking alternative routes. A suggestion as to why the assumed inflexibility is strictly tied to the use of the slow route, but does not occur after using the fast route, is that these routes could differ in their reliance on controlled processing. The fast route could represent an automatic processing route, which is plausible given that ample evidence exists on the automaticity of processing intact familiar symbols (e.g., LaBerge \& Samuels, 1974; Logan, 1978, 1988; Posner \& Snyder, 1975a). By contrast, the additional process that characterizes the slow route could be a controlled process, possibly engaged in separating relevant from irrelevant features, as suggested by Van Duren and Sanders (1988) or, alternatively, in searching for a familiar form in a noisy background. Whatever the activity of this additional process, the consequence of its invocation could be a temporary fixation on the slow route, which yields the subsequent cost-benefit characteristic. Similarly, the concurrence of costs with benefits has often been interpreted in the literature as an indicant of the involvement of attention. For instance, Posner and Snyder (1975b; see also Posner, 1978) inferred the attentional load of a mental pathway from the observation that benefits of a valid prime on subsequent processing concur with costs of an invalid prime. Research that explores the conjecture that the HSE reflects switching problems from controlled to automatic processing is in progress.

\section{REFERENCES}

ANDERSON, J. R. (1983). The architecture of cognition. Cambridge, MA: Harvard University Press.

BAMBER, D. (1969). Reaction times and error rates for "same" -"different" judgments of multidimensional stimuli. Perception \& Psychophysics, 6, 169-174.

FARELL, B. (1985). "Same"'-"different" judgements: A review of current controversies in perceptual comparisons. Psychological Review, 98, 419-456

HANSEN, W., \& Sanders, A. F. (1988). On the output of encoding during stimulus fixation. Acta Psychologica, 69, 95-107.

Houtmans, M. J. M., \& Sanders, A. F. (1983). Is information acquisition during large saccades possible? Bulletin of the Psychonomic Society, 21, 127-130.

Jonides, J., MACK, R. (1984). On the cost and benefit of cost and benefit. Psychological Bulletin, 96, 29-44.

KolERS, P. A. (1976). Reading a year later. Journal of Experimental Psychology: Human Learning \& Memory, 2, 554-565.

Kolers, P. A., \& Roediger, H. L., III (1984). Procedures of mind Journal of Verbal Learning \& Verbal Behavior, 23, 425-449.

Krueger, L. E. (1978). A theory of perceptual matching. Psychological Review, 85, 278-304.

Krueger, L. E. (1983). Probing Proctor's priming principle: The effect of simultaneous and sequential presentation on "same"'." different' judgements. Journal of Experimental Psychology: Learning, Memory, \& Cognition, 9, 511-523.

Krueger, L. E., \& Shapiro, R. G. (1981a). Intertrial effects of samedifferent judgements. Qkarterly Journal of Experimental Psychology, 33A, 241-265.

Krueger, L. E., \& Shapiro, R. G. (1981b). A reformulation of Proc- 
tor's unified theory for matching-task phenomena. Psychological Review, 88, 573-581.

LABERGE. D. \& S SAmuels. S. J. (1974). Toward a theory of automatic information processing in reading. Cognitive Psvchologv, 6, 293-323

LOGAN, G. D. (1978). Attention in character-classification tasks: Evidence for the automaticity of component stages. Journal of Experimen tal Psychology: General, 107, 32-63.

LOGAN, G. D. (1988). Toward an instance theory of automatization. Psychological Review, 95, 492-527.

Nickerson, R. S. (1975). Effects of correlated and uncorrelated noise on visual pattern matching. In P. M. A. Rabbit \& S. Dornic (Eds.) Attention and performance $V$ (pp. 655-668). New York: Academic Press.

NiEMI, P. (1979). Stimulus intensity effects on auditory and visual reaction processes. Acta Psychologica, 43, 299-312.

PoSNer, M. I. (1978). Chronometric explorations of mind. Hillsdale. NJ: Erlbaum.

Posner, M. I., \& SNyder, C. R. R. (1975a). Attention and cognitive control. In R. L. Solso (Ed.), Information processing and cognition: The Loyola symposium (pp. 55-85). Potomac, MD: Erlbaum.

Posner, M. I., \& SNyder, C. R. R. (1975b). Facilitation and inhibition in the processing of signals. In P. M. A. Rabbit \& S. Dornic (Eds.), Attention and performance $V$ (pp. 669-682). New York: Academic Press.

ProCTOR, R. W. (1981). A unified theory for matching-task phenomena. Psychological Review, 88, 291-326.

PROCTOR, R. W., \& RAO, K. V. (1983). Reinstating the original principles of Proctor's unified theory of matching-task phenomena: An evaluation of Krueger and Shapiro's reformulation. Psychological Review, 90, 21-37.

RoEdiger, H.L., III, \& BLAXTON, T. A. (1987). Effects of varying mo- dality, surface features, and retention interval on priming in wordfragment completion. Memory \& Cognition, 15, 379-388.

SANDERS, A. F. (1963). The selective process in the functional visual field. Assen: van Gorcum.

SANDERS, A. F. (1977). Structural and functional aspects of the reaction process. In S. Dornic (Ed.), Attention and performance VI (pp. 325). Hillsdale, NJ: Erlbaum.

SANDERS, A. F. (1980). Stage analysis of reaction processes. In G. E. Stelmach \& J. Requin (Eds.), Tutorials in motor behavior (pp. 331354). Amsterdam: North-Holland.

Sanders, A. F., \& Rath, A. (1991). Perceptual processing and speedaccuracy trade-off. Acta Psychologica, 77, 275-291.

Van der Molen, M.W., \& Keuss, P. J. G. (1981). Response selection and the processing of auditory intensity. Quarterly Journal of Experimental Psychology, 33A, 177-184.

VAN Duren, L., \& Sanders, A. F. (1988). On the robustness of the additive factors stage structure in blocked and mixed choice reaction designs. Acta Psychologica, 69, 83-94.

\section{NOTES}

1. The number of measurements per condition was $25 \%$ less in the mixed group than in the blocked group. This was due to the fact that the blocked and mixed conditions had originally been intended for two separate experiments instead of one.

2 . The author thanks an anonymous reviewer for suggesting this possibility.

(Manuscript received October 8, 1992; revision accepted for publication July 9, 1993.) 